\title{
Article
}

\section{Explicit, Determinantal, and Recurrent Formulas of Generalized Eulerian Polynomials ${ }^{\dagger}$}

\author{
Yan Wang ${ }^{1,2}\left(\mathbb{D}\right.$, Muhammet Cihat Dağli ${ }^{3}\left(\mathbb{D}\right.$, Xi-Min Liu $^{1}\left(\mathbb{D}\right.$ and Feng $Q i^{4,5, *}$ \\ 1 School of Mathematical Sciences, Dalian University of Technology, Dalian 116024, China; \\ yanwang_dlut@sina.com (Y.W.); ximinliu@dlut.edu.cn (X.-M.L.) \\ 2 College of Mathematics and Physics, Inner Mongolia University for Nationalities, Tongliao 028043, China \\ 3 Department of Mathematics, Akdeniz University, Antalya 07058, Turkey; mcihatdagli@akdeniz.edu.tr \\ 4 Institute of Mathematics, Henan Polytechnic University, Jiaozuo 454010, China \\ 5 School of Mathematical Sciences, Tianjin Polytechnic University, Tianjin 300387, China \\ * Correspondence: qifeng618@gmail.com \\ + Dedicated to Dr. Professor Peter S. Bullen retired from University of British Columbia in Canada.
}

check for

updates

Citation: Wang, Y.; Dağli, M.C.; Liu, X.-M.; Qi, F. Explicit, Determinantal, and Recurrent Formulas of Generalized Eulerian Polynomials. Axioms 2021, 10, 37. https://doi.org/ 10.3390 /axioms 10010037

Academic Editors: Clemente Cesarano and Hans J. Haubold

Received: 2 February 2021

Accepted: 16 March 2021

Published: 18 March 2021

Publisher's Note: MDPI stays neutral with regard to jurisdictional claims in published maps and institutional affiliations.

Copyright: (c) 2021 by the authors. Licensee MDPI, Basel, Switzerland. This article is an open access article distributed under the terms and conditions of the Creative Commons Attribution (CC BY) license (https:// creativecommons.org/licenses/by/ $4.0 /)$.
Abstract: In the paper, by virtue of the Faà di Bruno formula, with the aid of some properties of the Bell polynomials of the second kind, and by means of a general formula for derivatives of the ratio between two differentiable functions, the authors establish explicit, determinantal, and recurrent formulas for generalized Eulerian polynomials.

Keywords: Faà di Bruno formula; generalized Eulerian polynomial; Bell polynomial of the second kind; general formula for derivatives of the ratio between two differentiable functions; explicit formula; determinantal formula; recurrent formula; Stirling number of the second kind

MSC: primary 11B83; secondary 05A15; 11B73; 11C08; 11C20; $11 Y 55$

\section{Introduction}

Eulerian polynomials $A_{n}(t)$ for $n \geq 0$ can be generated ([1], p. 2) by

$$
\frac{1-t}{e^{u(t-1)}-t}=\sum_{n=0}^{\infty} A_{n}(t) \frac{u^{n}}{n !}, \quad t \neq 1
$$

and higher-order Eulerian polynomials $A_{n}^{(\alpha)}(t)$ can be generated ([2], p. 206) by

$$
\left[\frac{1-t}{e^{u(t-1)}-t}\right]^{\alpha}=\sum_{n=0}^{\infty} A_{n}^{(\alpha)}(t) \frac{u^{n}}{n !}, \quad t \neq 1,
$$

where $\alpha>0$. In [3], among other things, Eulerian polynomials $A_{n}(t)$ and higher-order Eulerian polynomials $A_{n}^{(\alpha)}(t)$ were expressed by

$$
A_{n}(t)=\sum_{k=0}^{n} k ! S(n, k)(t-1)^{n-k}
$$

and

$$
A_{n}^{(\alpha)}(t)=\frac{1}{\Gamma(\alpha)} \sum_{k=0}^{n} \Gamma(\alpha+k) S(n, k)(t-1)^{n-k},
$$

where $S(n, k)$ for $n \geq k \geq 0$ denotes the Stirling numbers of the second kind

$$
S(n, k)=\frac{1}{k !} \sum_{\ell=1}^{k}(-1)^{k-\ell}\left(\begin{array}{l}
k \\
\ell
\end{array}\right) \ell^{n}
$$


and can be generated by

$$
\frac{\left(e^{x}-1\right)^{k}}{k !}=\sum_{n=k}^{\infty} S(n, k) \frac{x^{n}}{n !} .
$$

See [2] (p. 202, Theorem A; p. 206, Theorem A) and [4].

In [5], among other things, it was proven that Eulerian polynomials $A_{n}(t)$ and higherorder Eulerian polynomials $A_{k}^{(\alpha)}(t)$ satisfy

$$
\sum_{k=0}^{n} \frac{s(n, k)}{(t-1)^{k}} A_{k}(t)=\frac{n !}{(t-1)^{n}}
$$

and

$$
\sum_{k=0}^{n} \frac{s(n, k)}{(t-1)^{k}} A_{k}^{(\alpha)}(t)=\frac{\Gamma(n+\alpha)}{\Gamma(\alpha)} \frac{1}{(t-1)^{n}}
$$

where $s(n, k)$, which can be generated (see [6,7]) by

$$
\frac{[\ln (1+x)]^{k}}{k !}=\sum_{n=k}^{\infty} s(n, k) \frac{x^{n}}{n !}, \quad|x|<1,
$$

stands for the Stirling numbers of the first kind. Formulas (1) and (4) are inversions of each other, while Formulas (2) and (5) are also inversions of each other.

In ([8], pp. 328-329), a determinantal expression

$$
A_{n}(t)=\left|\begin{array}{cccccccc}
1 & \frac{1}{1-t} & 0 & 0 & \ldots & 0 & 0 & 0 \\
0 & 1 & \frac{1}{1-t} & 0 & \ldots & 0 & 0 & 0 \\
0 & 1 & \left(\begin{array}{l}
2 \\
1
\end{array}\right) & \frac{1}{1-t} & \ldots & 0 & 0 & 0 \\
0 & 1 & \left(\begin{array}{c}
3 \\
1
\end{array}\right) & \left(\begin{array}{c}
3 \\
2
\end{array}\right) & \ldots & 0 & 0 & 0 \\
\ldots & \ldots & \ldots & \ldots & \ddots & \ldots & \ldots & \ldots \\
0 & 1 & \left(\begin{array}{c}
n-2 \\
1
\end{array}\right) & \left(\begin{array}{c}
n-2 \\
2
\end{array}\right) & \ldots & \left(\begin{array}{c}
n-2 \\
n-3
\end{array}\right) & \frac{1}{1-t} & 0 \\
0 & 1 & \left(\begin{array}{c}
n-1 \\
1
\end{array}\right) & \left(\begin{array}{c}
n-1 \\
2
\end{array}\right) & \ldots & \left(\begin{array}{c}
n-1 \\
n-3
\end{array}\right) & \left(\begin{array}{c}
n-1 \\
n-2
\end{array}\right) & \frac{1}{1-t} \\
0 & 1 & \left(\begin{array}{c}
n \\
1
\end{array}\right) & \left(\begin{array}{c}
n \\
n
\end{array}\right) & \cdots & \left(\begin{array}{c}
n-3 \\
n-3
\end{array}\right) & \left(\begin{array}{c}
n-2 \\
n-2
\end{array}\right) & \left(\begin{array}{c}
n \\
n-1
\end{array}\right)
\end{array}\right|, \quad n \geq 0
$$

and two recurrence relations

$$
\begin{aligned}
& A_{n}(t)=(t-1)^{n-1} \sum_{r=0}^{n-1}\left(\begin{array}{l}
n \\
r
\end{array}\right) \frac{A_{r}(t)}{(t-1)^{r}}, \quad n \in \mathbb{N}, \\
& A_{n}(t)=(-1)^{n+1}(t-1)^{n-1} t\left[1-t+\sum_{k=0}^{n-1}(-1)^{k}\left(\begin{array}{l}
n \\
k
\end{array}\right) \frac{A_{k}(t)}{(t-1)^{k}}\right], \quad n \geq 0
\end{aligned}
$$

were derived.

In [9], Eulerian polynomials $A_{n}(t)$ were generalized as $T_{n}(t, a, d)$, which can be generated ([9], Lemma 12) by

$$
\frac{(t-1) e^{a u(t-1)}}{t-e^{d u(t-1)}}=\sum_{n=0}^{\infty} T_{n}(t, a, d) \frac{u^{n}}{n !}
$$

The first four expressions of $T_{n}(t, a, d)$ are

$$
\begin{gathered}
T_{0}(t, a, d)=1, \quad T_{1}(t, a, d)=d+a(t-1), \\
T_{2}(t, a, d)=a^{2}(t-1)^{2}+2 a d(t-1)+d^{2}(t+1),
\end{gathered}
$$

and

$$
T_{3}(t, a, d)=a^{3}(t-1)^{3}+3 a^{2} d(t-1)^{2}+3 a d^{2}\left(t^{2}-1\right)+d^{3}\left(t^{2}+4 t+1\right) .
$$


In this paper, as in the papers [10-12], by virtue of the Faà di Bruno formula (see Lemma 1 below), with the help of two properties of the Bell polynomials of the second kind (see Lemmas 2 and 3 below), and by means of a general formula for derivatives of the ratio between two differentiable functions (see Lemma 4 below), we establish explicit, determinantal, and recurrent formulas for generalized Eulerian polynomials $T_{n}(t, a, d)$ (see Theorems $1-3$ below).

\section{Lemmas}

To prove our main results, we need the following lemmas.

Lemma 1 ([2] (pp. 134 and 139) and [13]). The Bell polynomials of the second kind, or say, partial Bell polynomials, denoted by $B_{n, k}\left(x_{1}, x_{2}, \ldots, x_{n-k+1}\right)$ for $n \geq k \geq 0$, are defined by

$$
B_{n, k}\left(x_{1}, x_{2}, \ldots, x_{n-k+1}\right)=\sum_{\substack{1 \leq i \leq n, \ell_{\ell} \in\{0\} \cup \mathbb{N} \\ \sum_{i=1}^{n} i \ell_{i}=n, \Sigma_{i=1}^{n} \ell_{i}=k}}^{\infty} \frac{n !}{\prod_{i=1}^{\ell-k+1} \ell_{i} !} \prod_{i=1}^{\ell-k+1}\left(\frac{x_{i}}{i !}\right)^{\ell_{i}} .
$$

The Faà di Bruno formula can be described in terms of the Bell polynomials of the second kind $B_{n, k} b y$

$$
\frac{\mathrm{d}^{n}}{\mathrm{~d} t^{n}} f \circ h(t)=\sum_{k=0}^{n} f^{(k)}(h(t)) B_{n, k}\left(h^{\prime}(t), h^{\prime \prime}(t), \ldots, h^{(n-k+1)}(t)\right) .
$$

Lemma 2 ([2] (p. 135) and [13]). For $n \geq k \geq 0$, we have

$$
B_{n, k}\left(a b x_{1}, a b^{2} x_{2}, \ldots, a b^{n-k+1} x_{n-k+1}\right)=a^{k} b^{n} B_{n, k}\left(x_{1}, x_{2}, \ldots, x_{n-k+1}\right),
$$

where $a$ and $b$ are complex numbers.

Lemma 3 ([2], p. 135). For $n \geq k \geq 0$, we have

$$
B_{n, k}(1,1, \ldots, 1)=S(n, k) .
$$

Lemma 4 ([14], p. 40, Entry 5). For $k \geq 0$ and two differentiable functions $p(x)$ and $q(x) \neq 0$, we have

$$
\frac{\mathrm{d}^{k}}{\mathrm{~d} x^{k}}\left[\frac{p(x)}{q(x)}\right]=\frac{(-1)^{k}}{q^{k+1}}\left|\begin{array}{cccccc}
p & q & 0 & \cdots & 0 & 0 \\
p^{\prime} & q^{\prime} & q & \cdots & 0 & 0 \\
p^{\prime \prime} & q^{\prime \prime} & \left(\begin{array}{c}
2 \\
1
\end{array}\right) q^{\prime} & \cdots & 0 & 0 \\
\vdots & \vdots & \vdots & \ddots & \vdots & \vdots \\
p^{(k-2)} & q^{(k-2)} & \left(\begin{array}{c}
k-2 \\
1
\end{array}\right) q^{(k-3)} & \cdots & q & 0 \\
p^{(k-1)} & q^{(k-1)} & \left(\begin{array}{c}
k-1 \\
1
\end{array}\right) q^{(k-2)} & \cdots & \left(\begin{array}{c}
k-1 \\
k-2
\end{array}\right) q^{\prime} & q \\
p^{(k)} & q^{(k)} & \left(\begin{array}{c}
k \\
1
\end{array}\right) q^{(k-1)} & \cdots & \left(\begin{array}{c}
k \\
k-2
\end{array}\right) q^{\prime \prime} & \left(\begin{array}{c}
k \\
k-1
\end{array}\right) q^{\prime}
\end{array}\right| .
$$

In other words, Formula (10) can be represented as

$$
\frac{\mathrm{d}^{k}}{\mathrm{~d} x^{k}}\left[\frac{p(x)}{q(x)}\right]=\frac{(-1)^{k}}{q^{k+1}(x)}\left|W_{(k+1) \times(k+1)}(x)\right|,
$$

where $\left|W_{(k+1) \times(k+1)}(x)\right|$ denotes the determinant of the matrix

$$
W_{(k+1) \times(k+1)}(x)=\left[U_{(k+1) \times 1}(x) \quad V_{(k+1) \times k}(x)\right]
$$


such that $U_{(k+1) \times 1}(x)$ has elements $u_{\ell, 1}(x)=p^{(\ell-1)}(x)$ for $1 \leq \ell \leq k+1$ and $V_{(k+1) \times k}(x)$ has entries of the form

$$
v_{i, j}(x)= \begin{cases}\left(\begin{array}{l}
i-1 \\
j-1
\end{array}\right) q^{(i-j)}(x), & i-j \geq 0 \\
0, & i-j<0\end{cases}
$$

for $1 \leq i \leq k+1$ and $1 \leq j \leq k$.

\section{Explicit, Determinantal, and Recurrent Formulas}

In this section, we establish explicit, determinantal, and recurrent formulas for generalized Eulerian polynomials $T_{n}(t, a, d)$.

Theorem 1. For $n \geq 0$, the generalized Eulerian polynomials $T_{n}(t, a, d)$ can be explicitly computed by

$$
T_{n}(t, a, d)=(t-1)^{n} \sum_{k=0}^{n}\left(\begin{array}{l}
n \\
k
\end{array}\right) d^{k} a^{n-k} \sum_{i=0}^{k} \frac{i !}{(t-1)^{i}} S(k, i) .
$$

Proof. This is the first proof. Applying the functions $f(v)=\frac{1}{v}$ and $v=g(u)=t-e^{d u(t-1)}$ to the Faà di Bruno Formula (7) and using the identities (8) and (9) yield that

$$
\begin{aligned}
\frac{\mathrm{d}^{k}}{\mathrm{~d} u^{k}}\left[\frac{1}{t-e^{d u(t-1)}}\right]= & \sum_{i=0}^{k}\left(\frac{1}{v}\right)^{(i)} B_{k, i}\left(-d(t-1) e^{d u(t-1)},-d^{2}(t-1)^{2} e^{d u(t-1)},\right. \\
& \left.\ldots,-d^{k-i+1}(t-1)^{k-i+1} e^{d u(t-1)}\right) \\
= & \sum_{i=0}^{k} \frac{(-1)^{i} i !}{v^{i+1}}(-1)^{i} d^{k}(t-1)^{k} e^{i d u(t-1)} B_{k, i}(1,1, \ldots, 1) \\
= & d^{k}(t-1)^{k} \sum_{i=0}^{k} \frac{i !}{\left[t-e^{d u(t-1)}\right]^{i+1}} e^{i d u(t-1)} S(k, i) .
\end{aligned}
$$

Hence, we obtain that

$$
\lim _{u \rightarrow 0} \frac{\mathrm{d}^{k}}{\mathrm{~d} u^{k}}\left[\frac{1}{t-e^{d u(t-1)}}\right]=d^{k}(t-1)^{k} \sum_{i=0}^{k} \frac{i !}{(t-1)^{i+1}} S(k, i) .
$$

On the other hand, it is easy to see that

$$
\lim _{u \rightarrow 0}\left[e^{a u(t-1)}\right]^{(k)}=\lim _{u \rightarrow 0}\left[a^{k}(t-1)^{k} e^{a u(t-1)}\right]=a^{k}(t-1)^{k} .
$$

Using Leibnitz's formula for the $n$th derivative of the product of two functions gives

$$
\begin{array}{r}
\frac{\mathrm{d}^{n}}{\mathrm{~d} u^{n}}\left[\frac{(t-1) e^{a u(t-1)}}{t-e^{d u(t-1)}}\right]=(t-1) \sum_{k=0}^{n}\left(\begin{array}{l}
n \\
k
\end{array}\right) \frac{\mathrm{d}^{k}}{\mathrm{~d} u^{k}}\left[\frac{1}{t-e^{d u(t-1)}}\right]\left[e^{a u(t-1)}\right]^{(n-k)} \\
=(t-1)^{n+1} \sum_{k=0}^{n}\left(\begin{array}{l}
n \\
k
\end{array}\right) d^{k} \sum_{i=0}^{k} \frac{i !}{\left[t-e^{d u(t-1)}\right]^{i+1}} e^{i d u(t-1)} S(k, i)\left[a^{n-k} e^{a u(t-1)}\right] .
\end{array}
$$

Accordingly, it follows that

$$
\begin{aligned}
\lim _{u \rightarrow 0} \frac{\mathrm{d}^{n}}{\mathrm{~d} u^{n}}\left[\frac{(t-1) e^{a u(t-1)}}{t-e^{d u(t-1)}}\right] & =\sum_{k=0}^{n}\left(\begin{array}{l}
n \\
k
\end{array}\right) d^{k}(t-1)^{k} \sum_{i=0}^{k} \frac{i !}{(t-1)^{i}} S(k, i) a^{n-k}(t-1)^{n-k} \\
& =(t-1)^{n} \sum_{k=0}^{n}\left(\begin{array}{l}
n \\
k
\end{array}\right) d^{k} a^{n-k} \sum_{i=0}^{k} \frac{i !}{(t-1)^{i}} S(k, i) .
\end{aligned}
$$


Considering the generating function (6) leads to (11). The proof of Theorem 1 is complete.

Proof. This is the second proof. Lemma 11 in [9] reads that

$$
T_{n}(t, a, d)=\sum_{j=0}^{n}\left(\begin{array}{l}
n \\
j
\end{array}\right) d^{j} A_{j}(t)(a t-a)^{n-j} .
$$

Substituting Formula (1) in ([3],Theorem 1) into the above formula results in the explicit Formula (11).

Proof. This is the third proof. By virtue of the generating function (3), it is easy to see that

$$
\begin{aligned}
& \frac{t-1}{t-e^{d u(t-1)}}=\frac{t-1}{t-1-\left[e^{d u(t-1)}-1\right]}=\frac{1}{1-\frac{e^{d u(t-1)}-1}{t-1}} \\
& =\sum_{i=0}^{\infty}\left[\frac{e^{d u(t-1)}-1}{t-1}\right]^{i}=\sum_{i=0}^{\infty} \frac{i !}{(t-1)^{i}} \sum_{k=i}^{\infty} S(k, i) \frac{[d u(t-1)]^{k}}{k !} .
\end{aligned}
$$

Then, it is not difficult to see that

$$
\begin{aligned}
\sum_{n=0}^{\infty} T_{n}(t, a, d) \frac{u^{n}}{n !} & =\frac{(t-1)}{t-e^{d u(t-1)}} e^{a u(t-1)} \\
& =\left[\sum_{i=0}^{\infty} \frac{i !}{(t-1)^{i}} \sum_{k=i}^{\infty} S(k, i) \frac{[d u(t-1)]^{k}}{k !}\right]\left[\sum_{i=0}^{\infty} \frac{[a u(t-1)]^{i}}{i !}\right] \\
& =\left[\sum_{i=0}^{\infty}\left(\sum_{j=0}^{i} S(i, j) \frac{j !}{(t-1)^{j}}\right) \frac{[d(t-1)]^{i} u^{i}}{i !}\right]\left[\sum_{i=0}^{\infty} \frac{[a(t-1)]^{i} u^{i}}{i !}\right] \\
& =\sum_{n=0}^{\infty}\left[(t-1)^{n} \sum_{k=0}^{n}\left(\begin{array}{l}
n \\
k
\end{array}\right) d^{k} a^{n-k} \sum_{i=0}^{k} \frac{i !}{(t-1)^{i}} S(k, i)\right] \frac{u^{n}}{n !} .
\end{aligned}
$$

Equating coefficients of $\frac{u^{n}}{n !}$ leads to the explicit Formula (11).

Remark 1. The second and third proofs of Theorem 1 are observed by two anonymous referees for avoiding direct and explicit differentiations.

Theorem 2. For $n \geq 0$, the generalized Eulerian polynomials $T_{n}(t, a, d)$ can be determinantally represented as

$$
\begin{aligned}
& T_{n}(t, a, d)= \\
& \left|\begin{array}{cccccc}
1 & -1 & 0 & \cdots & 0 & 0 \\
a(t-1) & d & -1 & \cdots & 0 & 0 \\
a^{2}(t-1)^{2} & d^{2}(t-1) & \left(\begin{array}{l}
2 \\
1
\end{array}\right) d & \cdots & 0 & 0 \\
\vdots & \vdots & \vdots & \ddots & \vdots & \vdots \\
a^{n-1}(t-1)^{n-1} & d^{n-1}(t-1)^{n-2} & \left(\begin{array}{c}
n-1 \\
1
\end{array}\right) d^{n-2}(t-1)^{n-3} & \cdots & \left(\begin{array}{c}
n-1 \\
n-2
\end{array}\right) d & -1 \\
a^{n}(t-1)^{n} & d^{n}(t-1)^{n-1} & \left(\begin{array}{c}
n \\
1
\end{array}\right) d^{n-1}(t-1)^{n-2} & \cdots & \left(\begin{array}{c}
n \\
n-2
\end{array}\right) d^{2}(t-1) & \left(\begin{array}{c}
n \\
n-1
\end{array}\right) d
\end{array}\right|
\end{aligned}
$$

Proof. Using Lemma 4 for $p(u)=(t-1) e^{a u(t-1)}$ and $q(u)=t-e^{d u(t-1)}$ gives 


$$
\begin{aligned}
& \frac{\mathrm{d}^{n}}{\mathrm{~d} u^{n}}\left[\frac{(t-1) e^{a u(t-1)}}{t-e^{d u(t-1)}}\right]=\frac{(-1)^{n}}{\left[t-e^{d u(t-1)}\right]^{(n+1)}} \mid \begin{array}{c}
(t-1) e^{a u(t-1)} \\
a(t-1)^{2} e^{a u(t-1)} \\
a^{2}(t-1)^{3} e^{a u(t-1)} \\
\vdots \\
a^{n-1}(t-1)^{n} e^{a u(t-1)} \\
a^{n}(t-1)^{n+1} e^{a u(t-1)}
\end{array} \\
& t-e^{d u(t-1)} \\
& -d(t-1) e^{d u(t-1)} \\
& -d^{2}(t-1)^{2} e^{d u(t-1)} \\
& t-e^{d u(t-1)} \\
& -\left(\begin{array}{l}
2 \\
1
\end{array}\right) d(t-1) e^{d u(t-1)} \\
& \begin{array}{c}
-d^{n-1}(t-1)^{n-1} e^{d u(t-1)} \\
-d^{n}(t-1)^{n} e^{d u(t-1)}
\end{array} \\
& -\left(\begin{array}{c}
n-1 \\
1
\end{array}\right) d^{n-2}(t-1)^{n-2} e^{d u(t-1)} \\
& -d^{n}(t-1)^{n} e^{d u(t-1)} \quad-\left(\begin{array}{l}
n \\
1
\end{array}\right) d^{n-1}(t-1)^{n-1} e^{d u(t-1)} \\
& \begin{array}{ccc|}
\ldots & 0 & 0 \\
\cdots & 0 & 0 \\
\cdots & 0 & 0 \\
\ddots & \vdots & \vdots \\
\cdots & -\left(\begin{array}{c}
n-1 \\
n-2
\end{array}\right) d(t-1) e^{d u(t-1)} & t-e^{d u(t-1)} \\
\cdots & -\left(\begin{array}{c}
n \\
n-2
\end{array}\right) d^{2}(t-1)^{2} e^{d u(t-1)} & -\left(\begin{array}{c}
n \\
n-1
\end{array}\right) d(t-1) e^{d u(t-1)}
\end{array}
\end{aligned}
$$

Therefore, we acquire

$$
\begin{aligned}
& \lim _{u \rightarrow 0} \frac{\mathrm{d}^{n}}{\mathrm{~d} u^{n}}\left[\frac{(t-1) e^{a u(t-1)}}{t-e^{d u(t-1)}}\right] \\
& =\frac{(-1)^{n}}{(t-1)^{(n+1)}} \mid \begin{array}{ccc}
t-1 & t-1 & 0 \\
a(t-1)^{2} & -d(t-1) & t-1 \\
a^{2}(t-1)^{3} & -d^{2}(t-1)^{2} & -\left(\begin{array}{l}
2 \\
1
\end{array}\right) d(t-1) \\
\vdots & \vdots & \vdots \\
a^{n-1}(t-1)^{n} & -d^{n-1}(t-1)^{n-1} & -\left(\begin{array}{c}
n-1 \\
1
\end{array}\right) d^{n-2}(t-1)^{n-2} \\
a^{n}(t-1)^{n+1} & -d^{n}(t-1)^{n} & -\left(\begin{array}{c}
n \\
1
\end{array}\right) d^{n-1}(t-1)^{n-1}
\end{array} \\
& \begin{array}{ccc|}
\cdots & 0 & 0 \\
\cdots & 0 & 0 \\
\cdots & 0 & 0 \\
\ddots & \vdots & \vdots \\
\cdots & -\left(\begin{array}{c}
n-1 \\
n-2
\end{array}\right) d(t-1) & t-1 \\
\cdots & -\left(\begin{array}{c}
n-2 \\
n-2
\end{array} d^{2}(t-1)^{2}\right. & -\left(\begin{array}{c}
n \\
n-1
\end{array}\right) d(t-1)
\end{array} \\
& =\left|\begin{array}{cccccc}
1 & -1 & 0 & \cdots & 0 & 0 \\
a(t-1) & d & -1 & \cdots & 0 & 0 \\
a^{2}(t-1)^{2} & d^{2}(t-1) & \left(\begin{array}{c}
2 \\
1
\end{array}\right) d & \cdots & 0 & 0 \\
\vdots & \vdots & \vdots & \ddots & \vdots & \vdots \\
a^{n-1}(t-1)^{n-1} & d^{n-1}(t-1)^{n-2} & \left(\begin{array}{c}
n-1 \\
1
\end{array}\right) d^{n-2}(t-1)^{n-3} & \cdots & \left(\begin{array}{c}
n-1 \\
n-2
\end{array}\right) d & -1 \\
a^{n}(t-1)^{n} & d^{n}(t-1)^{n-1} & \left(\begin{array}{c}
n \\
1
\end{array}\right) d^{n-1}(t-1)^{n-2} & \cdots & \left(\begin{array}{c}
n \\
n-2
\end{array}\right) d^{2}(t-1) & \left(\begin{array}{c}
n \\
n-1
\end{array}\right) d
\end{array}\right| .
\end{aligned}
$$

Considering the generating function (6) of $T_{n}(t, a, d)$, we finish the proof of Theorem

Theorem 3. For $n \geq 0$, the generalized Eulerian polynomials $T_{n}(t, a, d)$ possess the recurrence formula

$$
T_{n}(t, a, d)=a^{n}(t-1)^{n}+\sum_{k=0}^{n-1}\left(\begin{array}{l}
n \\
k
\end{array}\right) d^{n-k}(t-1)^{n-k-1} T_{k}(t, a, d),
$$


where an empty sum is understood to be 0.

Proof. This is the first proof. Since

$$
\left[t-e^{d u(t-1)}\right]\left[\frac{(t-1) e^{a u(t-1)}}{t-e^{d u(t-1)}}\right]=(t-1) e^{a u(t-1)},
$$

by differentiating $n$ times with respect to $u$ on both sides, we obtain

$$
\begin{aligned}
& \sum_{k=0}^{n}\left(\begin{array}{l}
n \\
k
\end{array}\right) \frac{\partial^{n-k}}{\partial u^{n-k}}\left[t-e^{d u(t-1)}\right] \frac{\partial^{k}}{\partial u^{k}}\left[\frac{(t-1) e^{a u(t-1)}}{t-e^{d u(t-1)}}\right] \\
= & {\left[t-e^{d u(t-1)}\right] \frac{\partial^{n}}{\partial u^{n}}\left[\frac{(t-1) e^{a u(t-1)}}{t-e^{d u(t-1)}}\right] } \\
& -\sum_{k=0}^{n-1}\left(\begin{array}{l}
n \\
k
\end{array}\right) d^{n-k}(t-1)^{n-k} e^{d u(t-1)} \frac{\partial^{k}}{\partial u^{k}}\left[\frac{(t-1) e^{a u(t-1)}}{t-e^{d u(t-1)}}\right] \\
= & a^{n}(t-1)^{n+1} e^{a u(t-1)} .
\end{aligned}
$$

Letting $u \rightarrow 0$ and taking into account the generating function (6) of generalized Eulerian polynomials $T_{n}(t, a, d)$ yield

$$
(t-1) T_{n}(t, a, d)-\sum_{k=0}^{n-1}\left(\begin{array}{l}
n \\
k
\end{array}\right) d^{n-k}(t-1)^{n-k} T_{k}(t, a, d)=a^{n}(t-1)^{n+1}
$$

which can be rewritten as (13). The proof of Theorem 3 is complete.

Proof. This is the second proof. Substituting the power series expansions

$$
t-e^{d u(t-1)}=t-\sum_{k=0}^{\infty} \frac{[d u(t-1)]^{k}}{k !}=t-1-\sum_{k=1}^{\infty}[d(t-1)]^{k} \frac{u^{k}}{k !}
$$

and (6) into the left-hand side of (14) yields

$$
\begin{aligned}
& {\left[t-e^{d u(t-1)}\right]\left[\frac{(t-1) e^{a u(t-1)}}{t-e^{d u(t-1)}}\right]=\left[t-1-\sum_{k=1}^{\infty}[d(t-1)]^{k} \frac{u^{k}}{k !}\right] \sum_{n=0}^{\infty} T_{n}(t, a, d) \frac{u^{n}}{n !} } \\
= & (t-1) \sum_{n=0}^{\infty} T_{n}(t, a, d) \frac{u^{n}}{n !}-\left[\sum_{k=0}^{\infty}[d(t-1)]^{k+1} \frac{u^{k+1}}{(k+1) !}\right] \sum_{n=0}^{\infty} T_{n}(t, a, d) \frac{u^{n}}{n !} \\
= & (t-1) \sum_{n=0}^{\infty} T_{n}(t, a, d) \frac{u^{n}}{n !}-\sum_{n=0}^{\infty}\left[\sum_{k=0}^{n} \frac{[d(t-1)]^{n-k+1}}{(n-k+1) !} \frac{T_{k}(t, a, d)}{k !}\right] u^{n+1} \\
= & (t-1) \sum_{n=0}^{\infty} T_{n}(t, a, d) \frac{u^{n}}{n !}-\sum_{n=0}^{\infty}\left[\sum_{k=0}^{n}\left(\begin{array}{c}
n+1 \\
k
\end{array}\right)[d(t-1)]^{n-k+1} T_{k}(t, a, d)\right] \frac{u^{n+1}}{(n+1) !} \\
= & (t-1) \sum_{n=0}^{\infty} T_{n}(t, a, d) \frac{u^{n}}{n !}-\sum_{n=1}^{\infty}\left[\sum_{k=0}^{n-1}\left(\begin{array}{l}
n \\
k
\end{array}\right)[d(t-1)]^{n-k} T_{k}(t, a, d)\right] \frac{u^{n}}{n !} \\
= & (t-1) T_{0}(t, a, d)+\sum_{n=1}^{\infty}\left[(t-1) T_{n}(t, a, d)-\sum_{k=0}^{n-1}\left(\begin{array}{l}
n \\
k
\end{array}\right)[d(t-1)]^{n-k} T_{k}(t, a, d)\right] \frac{u^{n}}{n !} .
\end{aligned}
$$

On the other hand, the right-hand side of (14) can be expanded as

$$
(t-1) e^{a u(t-1)}=\sum_{n=0}^{\infty} a^{n}(t-1)^{n+1} \frac{u^{n}}{n !} .
$$


Therefore, we obtain

$$
\begin{aligned}
(t-1) T_{0}(t, a, d)+\sum_{n=1}^{\infty}\left[(t-1) T_{n}(t, a, d)-\sum_{k=0}^{n-1}\left(\begin{array}{l}
n \\
k
\end{array}\right)[d(t-1)]^{n-k} T_{k}(t, a, d)\right] \frac{u^{n}}{n !} \\
=\sum_{n=0}^{\infty} a^{n}(t-1)^{n+1} \frac{u^{n}}{n !}
\end{aligned}
$$

Equating the coefficients of $\frac{u^{n}}{n !}$ arrives at $(t-1) T_{0}(t, a, d)=t-1$ and

$$
(t-1) T_{n}(t, a, d)-\sum_{k=0}^{n-1}\left(\begin{array}{l}
n \\
k
\end{array}\right)[d(t-1)]^{n-k} T_{k}(t, a, d)=a^{n}(t-1)^{n+1} .
$$

The recursive Formula (13) is thus proven.

Remark 2. The second proof of Theorem 3 is observed by an anonymous referee for avoiding direct and explicit differentiations.

\section{Conclusions}

The theory of polynomials is important in mathematics and mathematical sciences. Series expansions can be regarded as polynomials of infinite terms. Special polynomials such as the Bernoulli polynomials, the Euler polynomials, and the Stirling polynomials are particularly important and interesting. For studying a special sequence of polynomials, one aspect should be to discover its closed-form expressions or recurrent relations. Explicit and determinantal expressions are possibly two forms of closed-form expressions. Especially, finding determinantal expressions of special polynomials are generally difficult and interesting in mathematics. In recent years, the fourth author of this paper gave and applied a general, comparatively effective, comparatively easy, comparatively simple method to set up determinantal expressions and recursive relations of some special polynomials, including the above-mentioned famous Bernoulli numbers and polynomials [15], Euler numbers and polynomials [16,17], and Stirling numbers and polynomials [18], by considering their generating functions. In this paper, after the papers $[1,3,7,8]$ in which Eulerian polynomials $A_{n}(x)$ and higher order Eulerian polynomials $A_{n}^{(\alpha)}(t)$ were investigated, the authors of this paper further considered the generalization $T_{n}(t, a, d)$ in [9] of the sequence of Eulerian polynomials $A_{n}(x)$ and established explicit, determinantal, and recursive formulas of the generalized Eulerian polynomials $T_{n}(t, a, d)$. Concretely speaking, in this paper, by virtue of the Faà di Bruno formula (7), with the aid of the identities (8) and (9), and by means of a general formula (10) for derivatives of the ratio between two differentiable functions, we established an explicit formula (11), a determinantal formula (12), and a recurrent formula (13) for generalized Eulerian polynomials $T_{n}(t, a, d)$ generated in (6).

Author Contributions: Writing-original draft, Y.W., M.C.D., X.-M.L., and F.Q. All authors contributed equally to the manuscript. All authors have read and agreed to the published version of the manuscript.

Funding: This work was partially supported by the National Natural Science Foundation of China (grant No. 11901322), by the Natural Science Foundation of Inner Mongolia (grant No. No. 2019MS01007), and by the Foundation of the Research Program of Science and Technology at Universities of Inner Mongolia (grant No. NJZY20119), China.

Institutional Review Board Statement: Not applicable.

Informed Consent Statement: Not applicable.

Data Availability Statement: Data sharing is not applicable to this article as no new data were created or analyzed in this study. 
Acknowledgments: The authors are thankful to anonymous referees for their careful corrections and valuable comments on the original version of this paper.

Conflicts of Interest: The authors declare no conflicts of interest.

\section{References}

1. Barry, P. Eulerian polynomials as moments, via exponential Riordan arrays. J. Integer Seq. 2011, 14, 1-14.

2. Comtet, L. Advanced Combinatorics: The Art of Finite and Infinite Expansions; Revised and Enlarged Edition; D. Reidel Publishing Co.: Dordrecht, The Netherlands, 1974. [CrossRef]

3. Qi, F.; Guo, B.-N. Explicit formulas and recurrence relations for higher order Eulerian polynomials. Indag. Math. 2017, 28 , 884-891. [CrossRef]

4. Qi, F. Diagonal recurrence relations, inequalities, and monotonicity related to the Stirling numbers of the second kind. Math. Inequal. Appl. 2016, 19, 313-323. [CrossRef]

5. Qi, F.; Lim, D.; Guo, B.-N. Some identities related to Eulerian polynomials and involving the Stirling numbers. Appl. Anal. Discrete Math. 2018, 12, 467-480. [CrossRef]

6. Qi, F. Diagonal recurrence relations for the Stirling numbers of the first kind. Contrib. Discrete Math. 2016, 11, 22-30. [CrossRef]

7. Qi, F.; Guo, B.-N. A diagonal recurrence relation for the Stirling numbers of the first kind. Appl. Anal. Discrete Math. 2018, 12, 153-165. [CrossRef]

8. Qi, F. Determinantal expressions and recurrence relations for Fubini and Eulerian polynomials. J. Interdiscip. Math. 2019, 22, 317-335. [CrossRef]

9. Xiong, T.; Tsao, H.-P.; Hall, J.I. General Eulerian numbers and Eulerian polynomials. J. Math. 2013, 2013, 629132. [CrossRef]

10. Dağlı, M.C. Closed formulas and determinantal expressions for higher-order Bernoulli and Euler polynomials in terms of Stirling numbers. Rev. R. Acad. Cienc. Exactas Fís. Nat. Ser. A Mat. RACSAM 2021, 115, 32. [CrossRef]

11. Hu, S.; Kim, M.-S. Two closed forms for the Apostol-Bernoulli polynomials. Ramanujan J. 2018, 46, 103-117. [CrossRef]

12. Qi, F.; Guo, B.-N. Several explicit and recursive formulas for generalized Motzkin numbers. AIMS Math. 2020, 5, 1333-1345. [CrossRef]

13. Qi, F.; Niu, D.-W.; Lim, D.; Yao, Y.-H. Special values of the Bell polynomials of the second kind for some sequences and functions. J. Math. Anal. Appl. 2020, 491, 124382. [CrossRef]

14. Bourbaki, N. Functions of a Real Variable, Elementary Theory; Translated from the 1976 French original by Philip Spain; Elements of Mathematics (Berlin); Springer: Berlin, Germany, 2004. [CrossRef]

15. Qi, F.; Chapman, R.J. Two closed forms for the Bernoulli polynomials. J. Number Theory 2016, 159, 89-100. [CrossRef]

16. Qi, F.; Guo, B.-N. A determinantal expression and a recurrence relation for the Euler polynomials. Adv. Appl. Math. Sci. 2017, 16, 297-309.

17. Qi, F.; Guo, B.-N. Explicit formulas for special values of the Bell polynomials of the second kind and for the Euler numbers and polynomials. Mediterr. J. Math. 2017, 14, 140. [CrossRef]

18. Qi, F.; Guo, B.-N. A closed form for the Stirling polynomials in terms of the Stirling numbers. Tbilisi Math. J. 2017, 10, 153-158. [CrossRef] 